\title{
Numerical Modeling of Unsaturated Soils Problems
}

\author{
M.D. Fredlund
}

\begin{abstract}
Numerical modeling (finite element analyses) of saturated-unsaturated soils problems generally involves the solution of linear or nonlinear partial differential equations, PDEs. The soil properties for unsaturated soils usually take on a functional form that subsequently requires an iterative procedure to obtain a solution. Special numerical solution techniques are helpful (and in some cases necessary) in order to have confidence that the results of the numerical solution are accurate. The dynamic upgrade of the finite element mesh (and time steps) during the iterative solution process have proven to be of significant value in ensuring the proper convergence of the numerical solution. The unsaturated soil property functions are usually obtained through use of estimation procedures based on the measurement of the soil-water characteristic curve, SWCC. One or more estimation procedures have been proposed in the research literature for soil property functions for each physical process of interest in unsaturated soil mechanics. The numerical modeller must be aware of the relationship between the estimated soil property functions and the solution technique. Boundary conditions required when solving unsaturated soils problems often involve the assessment of moisture and thermal flux conditions computed from meteorological records. There are conditions and requirements that must be quantifiable when solving unsaturated soils problems. The estimation of the unsaturated soil property functions makes the solution of unsaturated soils problems more complex than those of saturated soils.
\end{abstract}

Keywords: numerical modeling, unsaturated soil mechanics, partial differential equations, finite element analysis, adaptive mesh refinement.

\section{Introduction}

There are several distinct differences between the modeling saturated soil mechanics problems and unsaturated soils problems (or saturated-unsaturated soils problems). This paper describes the differences between these two classes of problems and illustrates numerical modeling techniques that have been found to perform well when modeling problems involving unsaturated soils. The boundaries for unsaturated soil mechanics problems often involve the assessment of moisture (and thermal) fluxes that are related to climatic conditions. Solving unsaturated soils problems with realistic moisture flux boundary conditions has proven to be challenging (Fredlund \& Stianson, 2011). Ground surface, moisture flux boundary conditions constitute another common difference between solving saturated and unsaturated soils problems.

Numerical modeling of most saturated soils problems is quite straight forward because the soil properties can generally be assumed to be constant values. For example, transient water seepage problems may require the designation of the hydraulic conductivity, $k_{w}$ (coefficient of permeability), and the water storage coefficient of volume change, $m_{v}$. Similarly, other saturated soils problems also have soil properties that can be input as constant values. In other words, saturated soil modeling generally involves the input of fixed, designated soil properties. Unsaturated soils problems, on the other hand, generally involve the designation of soil properties that have the form of mathematical functions. Consequently, numerical modeling solutions be- come nonlinear and require an iterative procedure to obtain a solution. Stated another way, saturated soil mechanics can be viewed as linear soil mechanics problems with constant soil properties while unsaturated soil mechanics can be viewed as nonlinear soil mechanics problems.

One of the challenges in solving unsaturated soil mechanics problems is related to obtaining solutions that have converged to the "correct solution". This is particularly important when solving transient field problems where the solution from one time step affects subsequent mass balance calculations. Fortunately, computing techniques such as "automatic mesh refinement", AMF, have been developed to ensure that the solution of nonlinear partial differential equations can indeed converge to a "correct solution".

\section{Theoretical Differences in Formulations Between Saturated Soils and Unsaturated Soils}

Any physical process involving soils can be formulated as a boundary-value problem within a continuum mechanics framework. The procedure starts with the selection of a representative elemental volume, REV, to which the conservative laws of physics are applied (e.g., conservation of mass and/or conservation of energy). The physical laws of behaviour for the process under consideration are then substituted into the conservative field equations. The physical laws of behaviour will either include soil properties with constant values or mathematical functions that describe the manner in which the soil properties vary with re-

Murray D. Fredlund, PhD., CEO and President, SoilVision Systems Ltd., Saskatoon, SK, Canada. e-mail: murray@ soilvision.com.

Invited Article, no discussion. 
spect to another variable that is part of the solution. Soil properties for problems involving unsaturated soils generally fall into the latter category (i.e., mathematical functions). The end result is a nonlinear (or possibly a linear) partial differential equation that needs to be solved through use of a numerical modeling technique.

It is generally possible to formulate the behaviour of unsaturated soil processes in such a way that saturated soil processes are also embraced. The unsaturated soil formulations constitute a more general formulation and the saturated soil case becomes a special case that is simpler to solve. Consequently, a single generalized formulation can be used for solving problems involving saturated- unsaturated soil systems.

The primary difference between saturated soil numerical modeling and unsaturated soil numerical modeling lies in the fact that the unsaturated soil properties are in the form of a mathematical function that contains one of the variables for which a solution is being sought. Consequently, an assumed value must first be selected for the unsaturated soil properties. Once a solution is obtained for the primary variables being solved for, a check must be made to ensure that the correct unsaturated soil properties were used in obtaining the solution. If the correct unsaturated soil properties were not used in the solution, the soil properties must be up-dated and the solution repeated. This procedure sets up an iterative technique that may continue for $10 \mathrm{~s}$ and even $100 \mathrm{~s}$ of iterations. In the end, the solution may not be a "correct solution" unless certain conditions have been met during the iterative solution procedure. Special mesh refinement techniques (e.g., AMR) have proven to be particularly useful in not only ensuring convergence but also ensuring convergence to an accurate (or correct) solution.

\section{Moisture Flux Boundary Conditions}

The ground surface forms the uppermost boundary when solving many unsaturated soils problems. In some unsaturated soils cases it is possible to apply a hydraulic head or pressure boundary condition at the ground surface, however, in most situations it is more realistic to apply a moisture flux boundary condition based on climatic conditions. Rainfall (and snowfall) can be applied as a downward moisture flux at the ground surface. In the absence of precipitation, an upward moisture flux can be applied to represent evaporation (or evapotranspiration). A decision must also be made with regard to whether or not evaporation should be applied during rainfall conditions. It appears that the most commonly used assumption is to have the two processes (i.e., precipitation and evaporation) occur simultaneously. Figure 1 shows the components associated with calculating water balance at the ground surface. Each component of moisture movement must be evaluated, however, there is an interaction between components such as rainfall (intensity) and runoff.

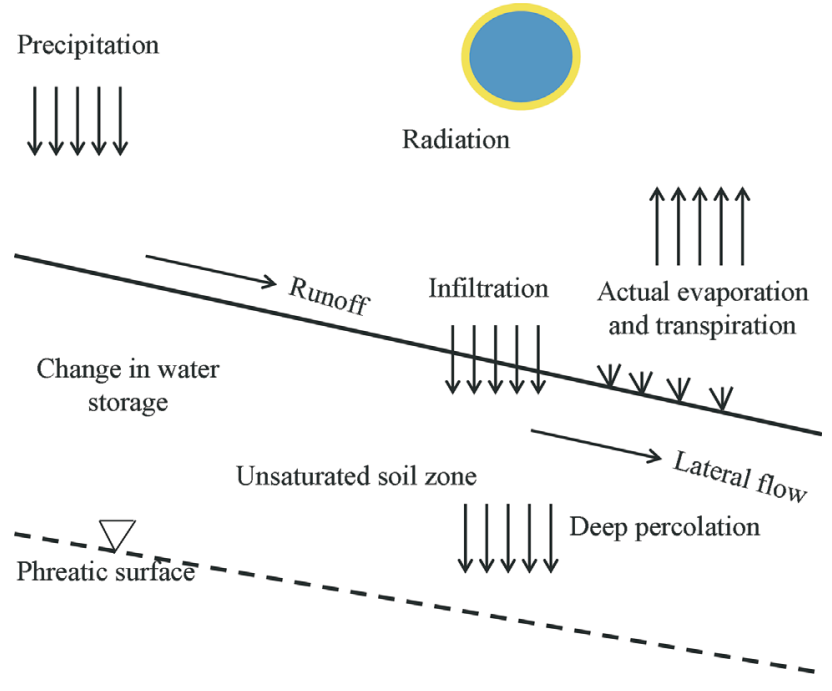

Figure 1 - Primary moisture components of a typical nearground-surface geotechnical engineering problem (Fredlund \& Stianson, 2011).

Meteorological stations commonly monitor precipitation on a daily basis. The daily precipitation records are valuable but it is the intensity of rainfall records that are of greatest significance in evaluating the interaction between infiltration and runoff. Figure 2 shows a typical weather station record of daily precipitation and the cumulative rainfall at a particular site in Canada. The graph shows that most of the rainfall occurs during the spring and fall seasons. The simulation of transient infiltration conditions requires the usage of short time steps often in the order of minutes. Consequently, assumptions are required regarding the rainfall duration (and therefore, the average rainfall intensity). The infiltration of moisture is closely related to rainfall intensity and the permeability of the near-groundsurface soils.

In northern climates such as found in Canada, additional assumptions must be made with regard to the snow sublimation, snowmelt and spring runoff. Figure 3 illustrates how each year can be subdivided into periods of varying degrees of moisture movement activity. For example, the period from point 1 to point 2 (or 3), can be referred to as the inactive period. The period from point 3 to 4 , is the snowmelt period with significant activity. The snowmelt period is followed by the active growing period. Assumptions regarding "how best to handle" each season of the year have been addressed in the literature (Stianson \& Fredlund, 2011).

Evaporation of moisture from the ground surface is closely related to net radiation conditions (temperature) and wind speed at ground surface. There are two terms commonly associated with the calculation of evaporation moisture fluxes; namely, potential evaporation, PE, and actual evaporation, AE. The physical processes associated 


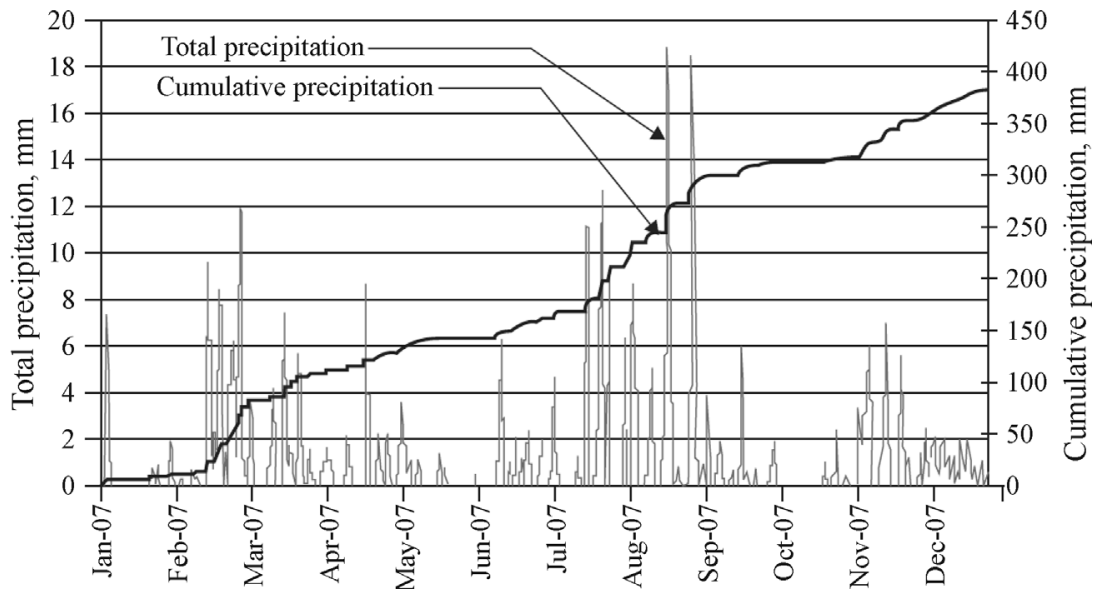

Figure 2 - Typical weather station record showing daily precipitation and cumulative rainfall at a particular site in Canada.

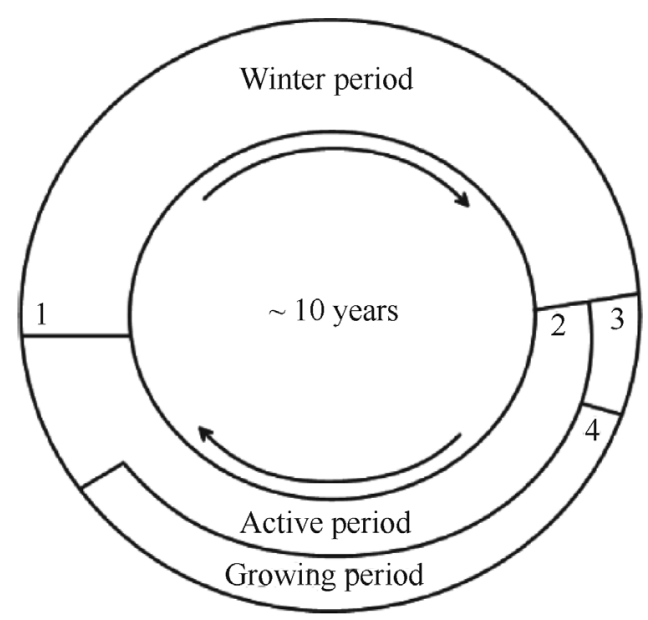

Figure 3 - Typical climatic periods of the year in cold climates (Fredlund \& Stianson, 2011).

with the PE term are quite well-known and the PE term can be calculated based on above-ground-surface conditions. Potential evaporation, PE, is dependent upon the vapour pressure gradient at ground surface, assuming there is an ample supply of water in the soil surface. Vapour pressure gradient is mainly a function of net radiation reaching the ground surface and the effect of "mixing" or wind turbulence at the ground surface. PE corresponds to the evaporation that occurs from a water saturated soil surface.

Actual evaporation, AE, is the moisture flux that is required for most numerical modeling scenarios. In addition to the effects of net radiation and "mixing", $\mathrm{AE}$ is also a function of the affinity of the ground surface soils for water (i.e., total suction) (Wilson et al., 1994). Total suction combines the effect of negative pore-water pressures (i.e., matric suction) and osmotic suction (i.e., effect of salts in the pore-water). There are other mechanisms that also significantly affect $\mathrm{AE}$ (Tran, 2013). For example, most of the salts in the pore-water are left behind to collect on the soil surface when water evaporates from a soil surface. The salt crust corresponds to a saturated salt solution with a high osmotic suction and a corresponding reduction in the evaporation rate.

In coarse-grained soils there is another factor affecting actual evaporation referred to as the "canopy effect". The canopy effect is usually associated with the vegetative cover on ground surface, however, desaturation of the soils near ground surface tends to produce another type of canopy effect (Tran, 2013). Most moisture movement occurs in the form of water vapour once the soil near ground surface dries to near residual water content conditions. At this point, water movement through evaporation is extremely slow.

Potential evaporation from a soil surface can be computed independent of the soil properties and a moisture flow model. The calculation of PE is relatively simple in comparison to the calculation for AE. The calculation of actual evaporative flux has proven to be a challenge in agriculture-related disciplines and geotechnical engineering. The calculation of actual evaporation requires that heat flow and moisture flow partial differential equations be combined and solved in either a coupled or uncoupled manner. The solution for $\mathrm{AE}$ is also undertaken in conjunction with the solution of the saturated-unsaturated moisture flow model for soil near ground surface.

Recent numerical computations of actual evaporation from sand columns show that it is possible to obtain reasonably reliable results provided the primary physical processes are taken into consideration (Fredlund et al., 2011; Tran, 2013). In particular, it is necessary to include a "canopy-type" effect when the water content of a relatively coarse material (e.g., sand) reduces to near residual water content conditions. The effect of salts in the pore-water and salt accumulation at the soil surface also affect the rate of evaporation from ground surface. The canopy effect and salts in the pore-water reduce actual evaporation from a soil surface by reducing the vapor pressure gradient at the soil 
surface. Figure 4 shows measured evaporation rates from a sand column (Wilson, 1990) along with a comparison with numerical simulations undertaken by Fredlund et al. (2011) and Tran (2013). The numerical simulations were performed using several procedures available in the SVFlux FlexPDEs software from SoilVision Systems Ltd.

\section{Finite Element Numerical Modeling}

The finite element numerical modeling procedure became available for usage as an engineering analysis tool after computers became available for rapidly solving large matrices of equations. Mainframe computers began to be used for numerical modeling in the late 1960s and in a matter of about one decade the computing power of these computers was available on desktop and laptop computers.

Seepage analyses were one of the first numerical solutions to find their way into geotechnical engineering practice. These problems had one degree of freedom at each node and many problems could be solved using head boundary conditions and zero flux boundary conditions (i.e., impervious boundaries). Solutions involving moisture flux boundary condition problems would prove to be considerably more challenging because of the need to combine heat and moisture partial differential equation solutions in order to calculate actual evaporation from ground surface.

Original finite element solutions in the 1960s involved the manual design of a finite element mesh that covered a cross-section of the soil continuum. Each element needed to be numbered. This exercise was followed by the

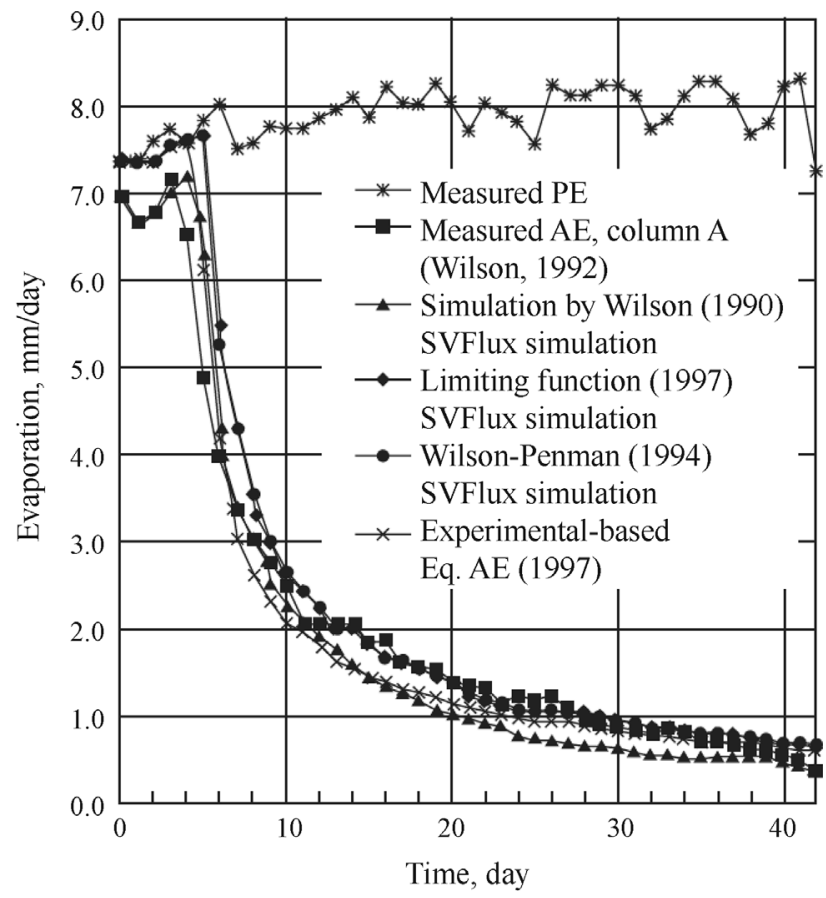

Figure 4 - Comparison of measured evaporation rates to predicted evaporation rates using coupled moisture movement models (Fredlund et al., 2012). numbering of the nodes around each element in a counter-clockwise manner. The time-consuming process of preparing a finite element mesh was later made easy through the use of the automatic design and numbering of elements and nodes comprising a finite element mesh.

The geotechnical engineer was aware that smaller elements needed to be used in regions where the hydraulic gradients were greater; however, there appeared to be no fixed rules with regard to the exact size of the small elements relative to the remainder of the finite element mesh. In other words, the design of the finite element mesh was largely a trial and error exercise. The relative sizes of elements throughout the soil continuum became of increased importance as attempts were made to solve nonlinear problems involving unsaturated soils. Unsaturated soils often had hydraulic conductivity values that could range over several orders of magnitude depending upon the suction in the soil.

The solution of unsaturated soils problems involved the solution of nonlinear partial differential seepage equations. Convergence to an accurate solution required that the finite element mesh be designed in a manner that met certain criteria for convergence. Research in the mathematics and computing science disciplines discovered that it was possible to design the finite element mesh in such a way that convergence was ensured (Oberkampf et al., 1995; Reddy, 2006; Roache, 2009). The methodology for the design of the finite element mesh resulted in removing the tedious and challenging task of designing a finite element mesh from the geotechnical engineer to the mathematician. The finite element design technique became known as "automatic adaptive mesh refinement".

The finite element mesh could be designed at the start of solving the problem and then changed, as necessary, as the solution moved towards convergence. For transient problems this meant that the finite element mesh could be changed from one time step to another time step, and also within each time step. The time steps could also be altered to assist in solving convergence issues. Finite element codes for solving geotechnical engineering problems can presently be categorized as: i.) manual mesh design codes, ii.) semi-automatic (or user-controlled) mesh design and iii.) automatic and adaptive mesh refinement, AMR, codes. Semi-automatic mesh generation codes have proven to be time-consuming and are quite often error-prone. Difficulties are most often encountered in situations where the mesh requires varying levels of change from one location to another location.

\subsection{Automatic adaptive mesh refinement, $A M R$}

The use of fully-automatic and adaptive mesh refinement codes are particularly useful in solving problems involving unsaturated soils. Two automatic adaptive mesh refinement, AMR, models are used in this paper to illustrate how converged solutions can be obtained with the assis- 
tance of automatic adaptive mesh refinement. The automated adaptive mesh refinement can reduce modeling time as well as errors during the modeling process. The AMR solutions were performed using the SVFlux - FlexPDE software. The AMR results are discussed in the contexts of the solutions for a series of seepage problems published by Chapuis (2012) using a user-controlled mesh design.

\subsection{Types of errors that occur in finite element analysis}

The mathematical type of errors that can be introduced into a finite element solution of a differential equation can be attributed to three basic sources (Reddy, 2006):

1. Domain approximation errors which are due to an approximation of actual domain,

2. Quadrature and finite arithmetic errors which are errors associated with the numerical evaluation of integrals and the numerical computations on the computer,

3. Approximation errors which are due to the approximation of the solution through interpolation functions.

The above list does not give consideration to errors in programming, and differences between the numerical model and the characterization of other physical processes (Oberkampf et al., 1995; Roache, 2009).

\subsection{Convergence of the solution}

The primary question that needs to be addressed when undertaking numerical modeling is, "How well does the model approximate the physical processes being studied and how well does the solution approach an accurate solution?" The answer to this question presumes that there is such a thing as an accurate solution to the problem. Studies involving interpolation theory can assist in understanding the meaning of an accurate solution to the problem.

\subsection{Finite element adaptive mesh refinement, $A M R$}

An adaptive mesh refinement, AMR, procedure measures the adequacy of the mesh during every iterative solution and refines the mesh wherever the estimated error is deemed to be too large. The mesh is refined and solution continues to iterate towards a solution until a user-defined "error tolerance" is achieved. The most common convergence criterion to use involves prescribing a total limit of the estimated error computed for the energy norm. Often this estimated energy norm error is specified to not exceed a specified percentage of the total norm of the solution. An adaptive mesh refinement procedure is used between iterations to reduce the estimated errors. This procedure is repeated until a solution has been obtained. The procedure is referred to as "adaptive" since the process depends on previous results at all stages.

There are various procedures for the refinement of the finite element solution. The refinement procedures can be broadly placed into two categories (Zienkiewicz et al., 2005).
1. The $h$-refinement involves the ongoing usage of the same class of element while the size of the element is changed. In some locations the elements will be made larger while in other locations the elements will be made smaller. The intent is to provide the maximum efficiency in reaching the desired solution,

2 . The $p$-refinement involves the ongoing usage of the same element size while there is a simple hierarchical increase in the order of the polynomial used in the definition of the elements.

It is sometimes useful to divide the above categories into sub-classes since the $h$-refinement can be applied in different ways. There are three typical $h$-refinement methods that can be described as follows:

1. Element subdivision can be used if the existing elements show too large an estimated error. The elements are simply divided into smaller elements while keeping the original element geometry boundaries intact,

2. Mesh regeneration (i.e., re-meshing) can be performed on the basis of a given solution. In this case, a new element size is predicted in all the domains and a new mesh is generated,

3. $r$-refinement involves keeping the total number of nodes constant while adjusting the position of the nodes to obtain an optimal approximation. This method may be difficult to use in practice and is therefore not the preferred procedure.

The $p$-refinement subclasses can be described as follows:

1. The polynomial order of each element is increased uniformly throughout the entire domain,

2. The polynomial order is increased locally while using hierarchical refinement.

Occasionally it is possible to combine the $h$ - and $p$ refinements and in this case the procedure is referred to as the $h p$ - refinement. The element size and the polynomial degree, $p$ are both altered when using the $h p$ - refinement procedure.

\section{Nonlinearity of the Unsaturated Material Properties}

The nonlinearity of the partial differential equations that need to be solved for unsaturated soils is related to the nonlinear soil property functions that define the unsaturated soil properties. The nonlinearity in unsaturated soil properties result from significant changes in the degree of saturation of the soil as a particular physical process is simulated. The degree of saturation is primarily related to changes in soil suction.

Hydraulic properties such as the coefficient of permeability and water storage of a soil can be computed as a function of matric suction in the range of suctions from zero to about $1500 \mathrm{kPa}$. The relationship between soil suction and the amount of water in the soil (e.g., volumetric water content and degree of saturation) has given rise to a series 
of estimation procedures that can be used to approximate the unsaturated soil property functions.

The rate of actual evaporation from a soil, on the other hand, is related to the total suction of the soil (i.e., matric suction plus osmotic suction).

\section{Prominent Role of the Soil-Water Characteristic Curve, SWCC}

The direct laboratory measurement of unsaturated soil property functions has proven to be too costly for most engineering projects. However, a variety of estimation procedures have been proposed and found to be of reasonable accuracy for most geotechnical engineering problems. The estimation procedures are based on the saturated soil properties along with information gleaned from the soil-water characteristic curve, SWCC. Various mathematical procedures have been applied to the SWCC (e.g., various forms of integration along the SWCC, differentiation of the SWCC, and direct use of the SWCC equation), to obtain the desired unsaturated soil property functions.

It appears that the unsaturated soil property functions associated with every physical process common to geotechnical engineering are quite nonlinear in form. Each unsaturated soil property function has been found to be related to the soil-water characteristic curve, SWCC. There is a drying and a wetting SWCC and as a result the unsaturated soil property functions are hysteretic. The geotechnical engineer must either use the drying SWCC or an approximation of the wetting and drying SWCCs to determine a suitable approximation of unsaturated soil properties.

Figure 5 shows typical drying and wetting branches of the soil-water characteristic curve, SWCC, for a sand soil. Water storage in a soil is defined as the change in the volume of water in the soil for a particular change in soil suction. Consequently, the SWCC (i.e., drying or wetting
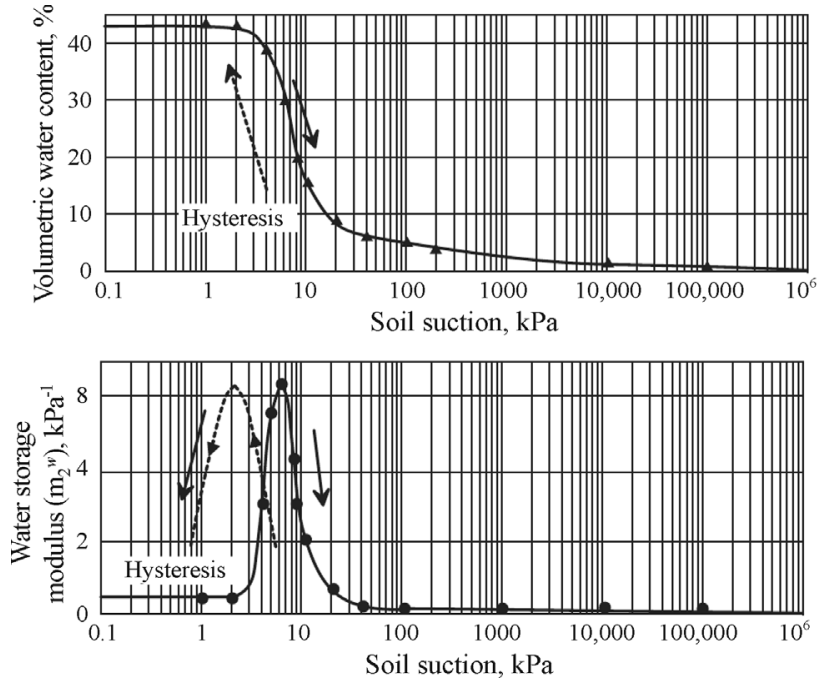

Figure 5 - Typical drying and wetting branches of the soil-water characteristic curve, SWCC, for a sand soil along with the corresponding water storage functions.

branches of volumetric water content $v s$. soil suction), can be differentiated to obtain the water storage function.

The hydraulic conductivity (or permeability) function can be estimated by starting with the saturated coefficient of permeability and integrating along the SWCC. Several integration procedures have been proposed and as a result there are several procedures for estimating the permeability function (Fredlund et al., 2012).

Figure 6 shows a water permeability function estimated for the sand soil with the drying SWCC shown in Fig. 5. At high soil suctions (i.e., approaching residual suction and beyond) the flow of liquid water become small and moisture movement is essentially through water vapor flow.

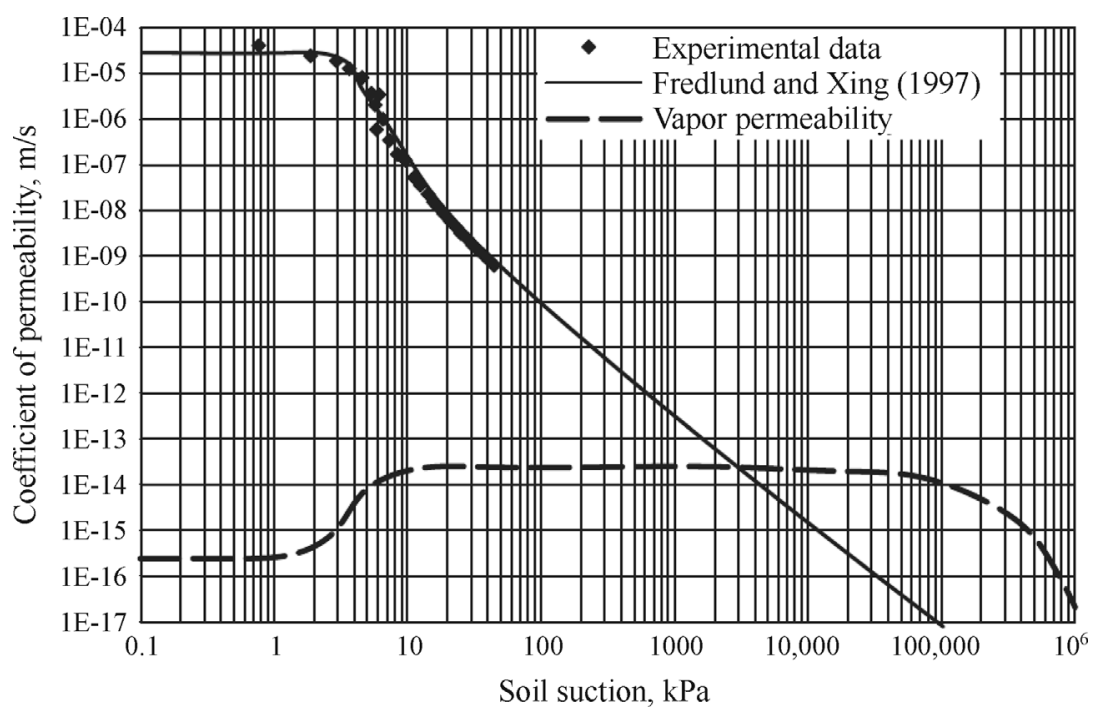

Figure 6 - Water permeability function estimated from the saturated coefficient of permeability and the soil-water characteristic curve. 


\section{Examples of Unsaturated Soils Problems}

Partial differential equations can be written to describe a wide range of physical processes that may be encountered in geotechnical engineering practice. The partial differential equations are nonlinear in character when an unsaturated soil is involved. Water flow through an unsaturated soil is used to illustrate how nonlinear partial differential equations can be solved to answer question asked of practicing geotechnical engineers.

The partial differential equation for water flow through an unsaturated soil can be written as follows (Fredlund et al., 2012).

$$
\begin{aligned}
& k_{w} \frac{\partial^{2} h_{w}}{\partial x^{2}}+\frac{\partial k_{w}}{\partial x} \frac{\partial h_{w}}{\partial x}+k_{w} \frac{\partial^{2} h_{w}}{\partial y^{2}}+ \\
& \frac{\partial k_{w}}{\partial y} \frac{\partial h_{w}}{\partial y}=m_{2}^{w} \rho_{w} g \frac{\partial h_{w}}{\partial t}
\end{aligned}
$$

where $h_{w}=$ hydraulic head $(\mathrm{m}), k_{w}=$ coefficient of permeability $(\mathrm{m} / \mathrm{s}), m_{w}^{2}=$ water storage coefficient, $(1 / \mathrm{kPa})$, $\rho_{w}=$ density of water, $g=$ acceleration due to gravity, $\left(\mathrm{m} / \mathrm{s}^{2}\right)$, $t=$ time (s) and $x, y=x$-coordinate and $y$-coordinate, respectively.

Equation 1 describes the physical process of water flow through a two-dimensional, unsaturated soil continuum. The equation applies for transient flow as well as steady state flow. The right-hand side of Eq. 1 becomes zero in the case of steady-state flow. The equation also applies for saturated soils. The difficulty in solving the partial differential equation arises when the soil is unsaturated because in this case there are three unknowns in this single equation; namely, $h_{w}, k_{w}$, and $m_{w}^{2}$. It is necessary to make use of two unsaturated soil property functions to render the problem determinate. A converged solution is obtained through use of an iterative process involving estimated values and repeated solutions. It is the adaptive mesh refinement, AMR, technique that assists in obtaining convergence to the "correct" solution.

Partial differential equations can also be written for other physical processes. The partial differential equation for air flow has similarities to the water flow equation with the exception that the permeate fluid is compressible and the unit weight is small. The air phase properties required when solving transient problems consists of: i.) the air permeability function, ii.) the air storage function, and iii.) the air compressibility function. In addition, the properties of air are quite sensitive to changes in temperature and as a result it might be necessary to combine heat and air flow partial differential equations when solving some engineering problems.

The heat flow partial differential equation for saturated-unsaturated soils requires soil property functions for: i.) the thermal conductivity function, and ii.) the heat storage function. Water can exist in three possible phases and as a result latent heat of fusion must also be taken into account when phase change is considered. The thermal properties are a function of the degree of saturation of the soil; however, many thermal conductivity problems can be solved through use of selected constants for the thermal properties. In other words, "coupling" between heat and moisture flow may not be required in some situations.

Stress analyses involving unsaturated soils also take on the form of partial differential equations for both saturated and unsaturated soils. There are two degrees of freedom at each node for a two-dimensional analysis. The stress analysis is often decoupled from the pore-fluid analysis; however, this may not be appropriate in some cases.

There are numerous other physical processes in saturated-unsaturated soil systems that can be described in the form of a partial differential equation. For example, chemicals move through the pore fluid system through the physical processes of advection, diffusion and adsorption. Advection flow can involve a coupling between pore fluid flow and chemical transport.

Unsaturated soils are generally encountered near ground surface in the region where several physical processes may overlap. Consequently, the combining of more than one process can be necessary to provide an adequate geotechnical engineering solution. Combining two or more physical processes can either be performed in a "coupled" or "uncoupled" manner. Modeling the combination of more than one physical process is presently an area of intensive research both in unsaturated soil mechanics as well as in other engineering disciplines.

\subsection{Use of water seepage analysis for unsaturated soils}

The flow of water through a homogeneous earth-fill dam is used as an example to illustrate the manner in which unsaturated soil properties are input in order to obtain a solution. Also illustrated is the type of output information that is typically shown in graphical form.

The soil-water characteristic curve, SWCC, must be known in order to estimate the unsaturated soil property functions (Fig. 7). The assumption is made that the soil does not undergo significant volume change as soil suction is increased. The solution of a steady-state type analysis only requires that the permeability function be known for the soil.

The permeability function is computed using an integration process along the SWCC, starting at the saturated coefficient of permeability (Fredlund et al., 2012; Fig. 8). The solution of a transient type analysis requires that the water storage function for the soil also be known (i.e., water storage vs. soil suction). The water storage function is obtained by differentiating the SWCC (Fig. 9).

The results of a transient analysis are shown by raising the water level along the upstream side of the reservoir of the dam from zero to $10 \mathrm{~m}$. Figure 10a shows the automatically generated finite element mesh along with the 


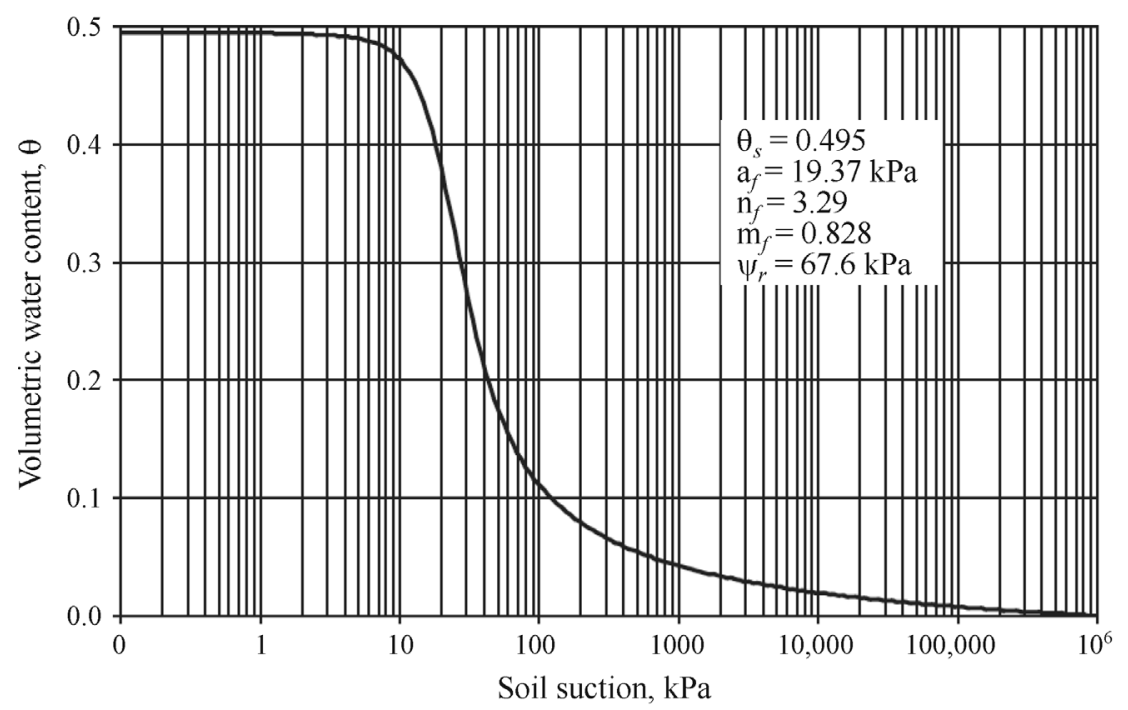

Figure 7 - Soil-water characteristic curve used to compute the unsaturated soil property functions for the example problem.

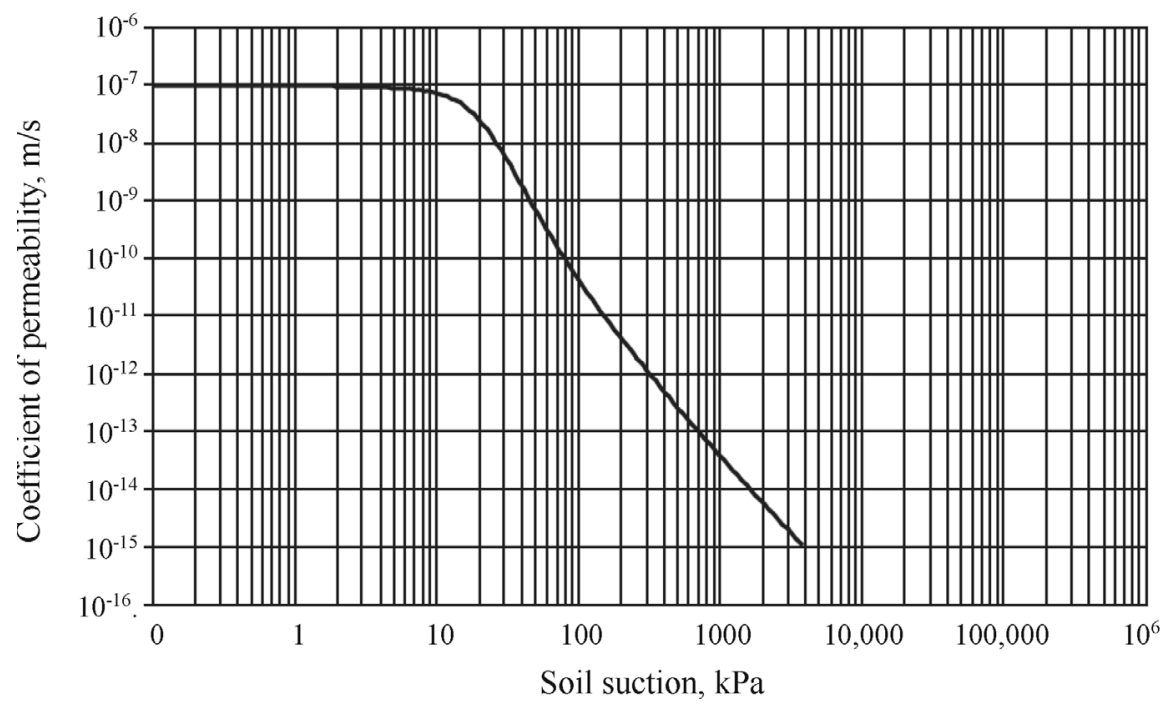

Figure 8 - Computed permeability function for the example problem.

head contours illustrating the dissipation of the head across the dam after 25 days. The finite element mesh is automatically refined in regions of higher hydraulic gradient to ensure convergence to an accurate solution.

Figure $10 \mathrm{~b}$ shows the same hydraulic head contours along with velocity vectors. The closeness of the head contours and the congestion of the velocity vectors are indicative of the high degree of nonlinearity of the unsaturated soil properties. It can be observed that the water velocity vectors move across the phreatic line (i.e., the line of zero pore-water pressure). Also noteworthy is the manner in which the head contours smoothly extend across the phreatic line to ground surface.

If the transient seepage analysis is extended for an elapsed time of 1500 days, the solution approaches that of a steady-state analysis where the water storage function is not required as input information. Figure 11a shows the automatically generated finite element mesh along with the hydraulic head contours corresponding to an elapsed time of 1500 days. Figure $11 \mathrm{~b}$ shows the hydraulic head contours along with velocity vectors. Once again there is some movement of water across the phreatic surface. In other words, the phreatic line does not constitute an uppermost flow line of seepage.

One further saturated-unsaturated seepage analysis is shown where the cross-section of a dam consisting of two materials, a shell with a low permeability core that does not extend to the surface of the dam. The results of a steadystate analysis of the dam are shown in Figs. 12a and 12b. Figure 12a shows the close spacing of the head contours across the core of the dam. Figure $12 \mathrm{~b}$ shows the velocity vectors with water moving over-top of the low permeability 


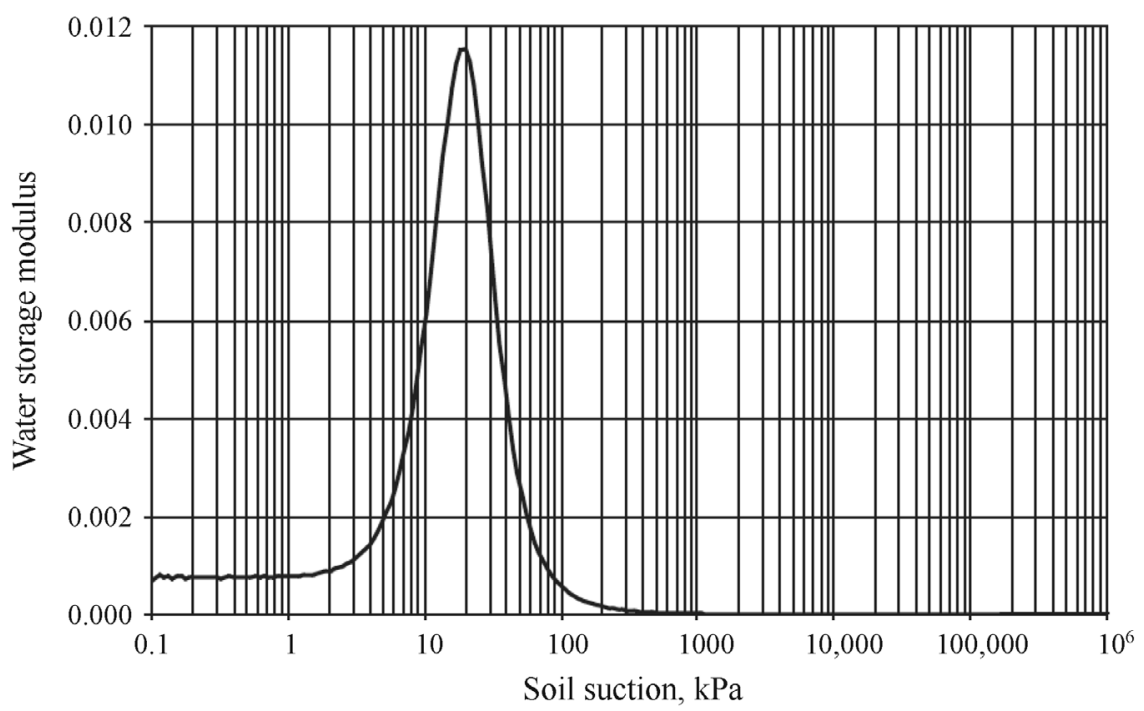

Figure 9 - Computed water storage function for the example problem.

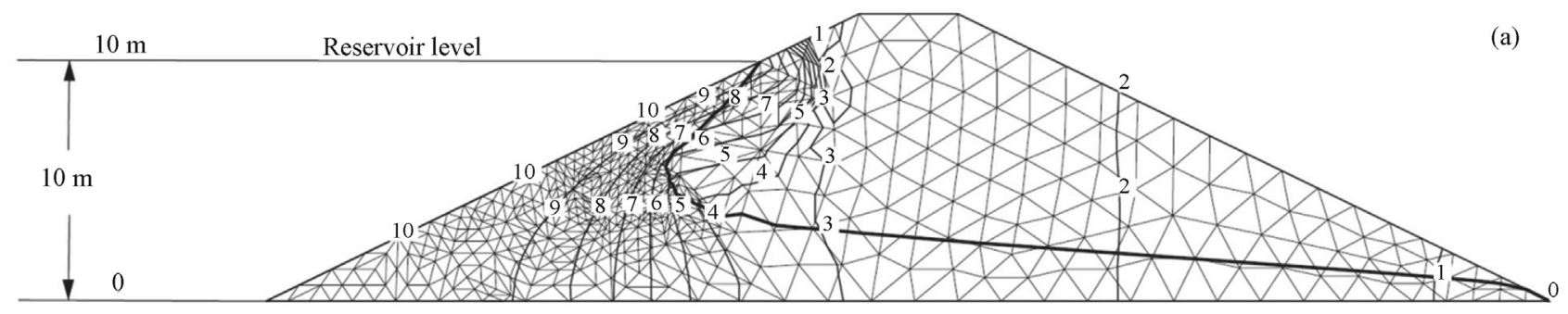

Time $=25$ days

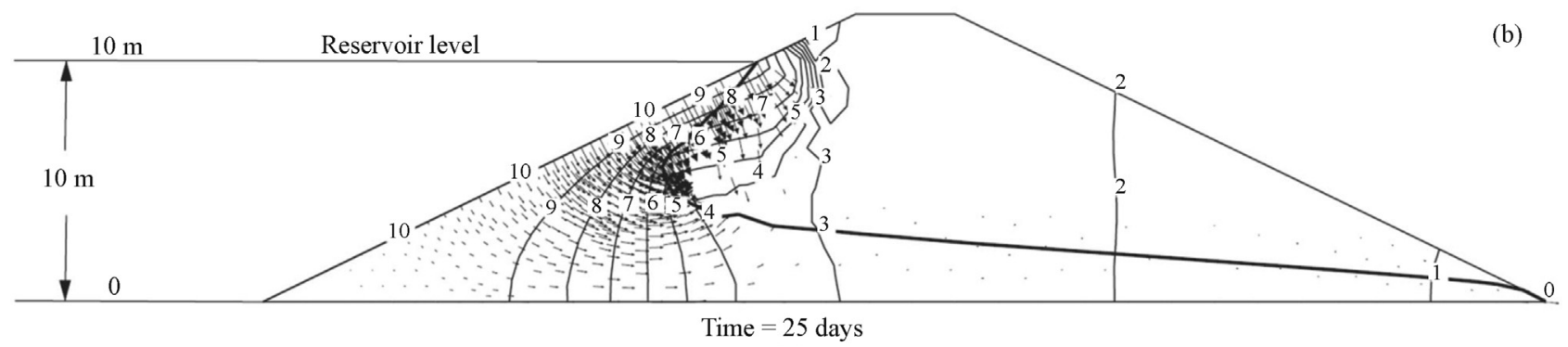

Figure 10 - a) Finite element mesh and hydraulic heads after the reservoir level was raised for 25 days. b) Velocity vectors and hydraulic heads after the reservoir level was raised for 25 days.

core through a syphon effect. The results indicate that it is easier for the water to flow upward through the unsaturated soil rather than to flow through the lower permeability core material. The siphon phenomenon was one of the early unsaturated soils that intrigued geotechnical engineers but is now readily explainable through use of saturated-unsaturated seepage analysis (Terzghi, 1943).

\section{Use of Automatic Adaptive Mesh Refinement for Saturated Seepage Examples}

Automatic adaptive mesh generation and refinement can also be used to advantage when modeling saturatedunsaturated seepage problems. Two example problems in- volving seepage through a saturated soil were analyzed by Chapuis (2012). These examples are re-analyzed taking advantage of automatic mesh generation and refinement. The example problems were solved using automatic mesh refinement, AMR, programmed in the SVFlux - FlexPDE finite element code.

\subsection{Cut-off example}

The geometry of the model dam analyzed is shown in Fig. 13. The dam has a partial cut-off wall and the soil has a saturated coefficient of permeability of $8.13 \times 10^{-3} \mathrm{~m} /$ day.

Chapuis (2012) obtained converged solutions using a uniform mesh with an element size of $0.5 \mathrm{~m}$. Figure 13 

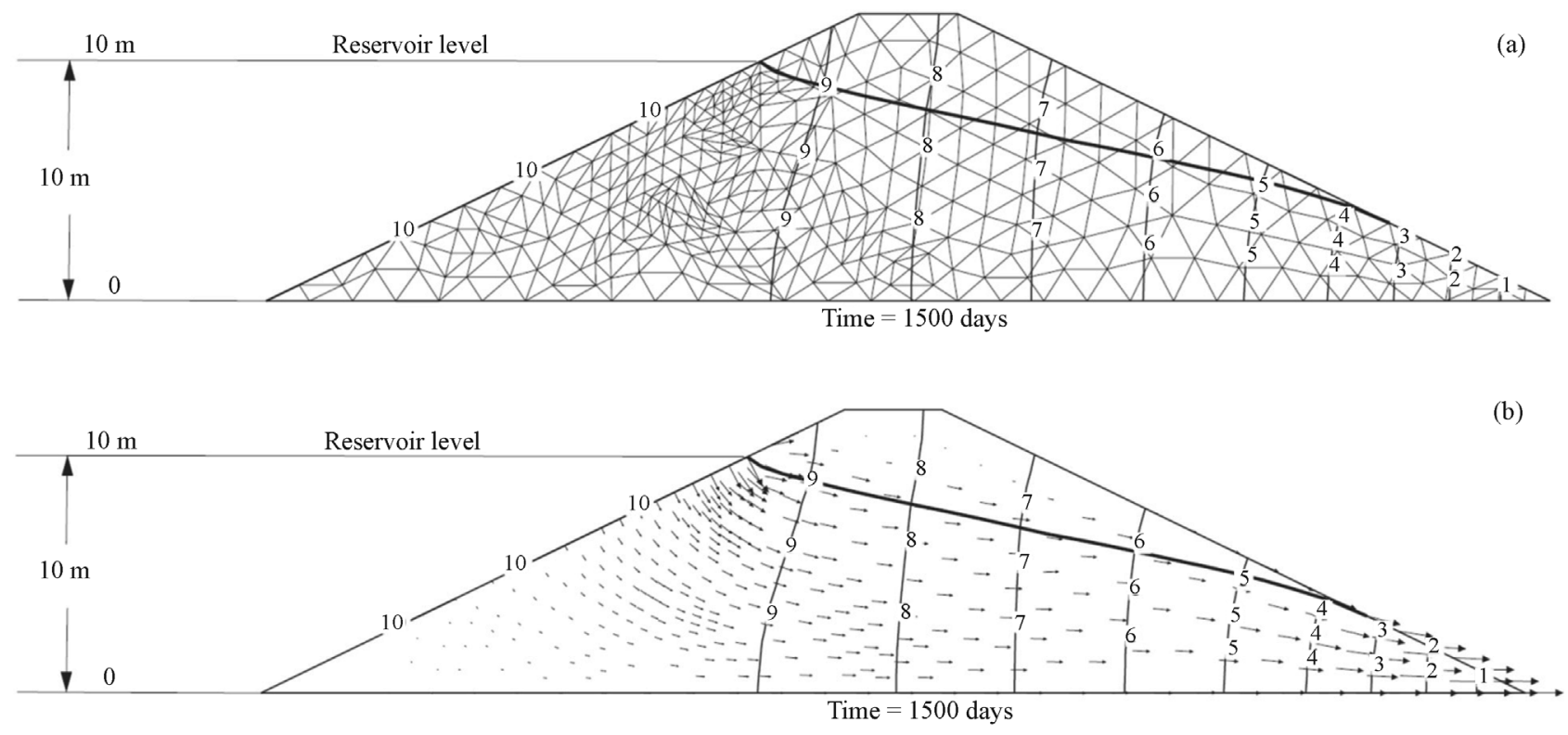

Figure 11 - a) Finite element mesh and hydraulic heads after the reservoir level was raised for 1500 days. b) Velocity vectors and hydraulic heads after the reservoir level was raised for 1500 days.
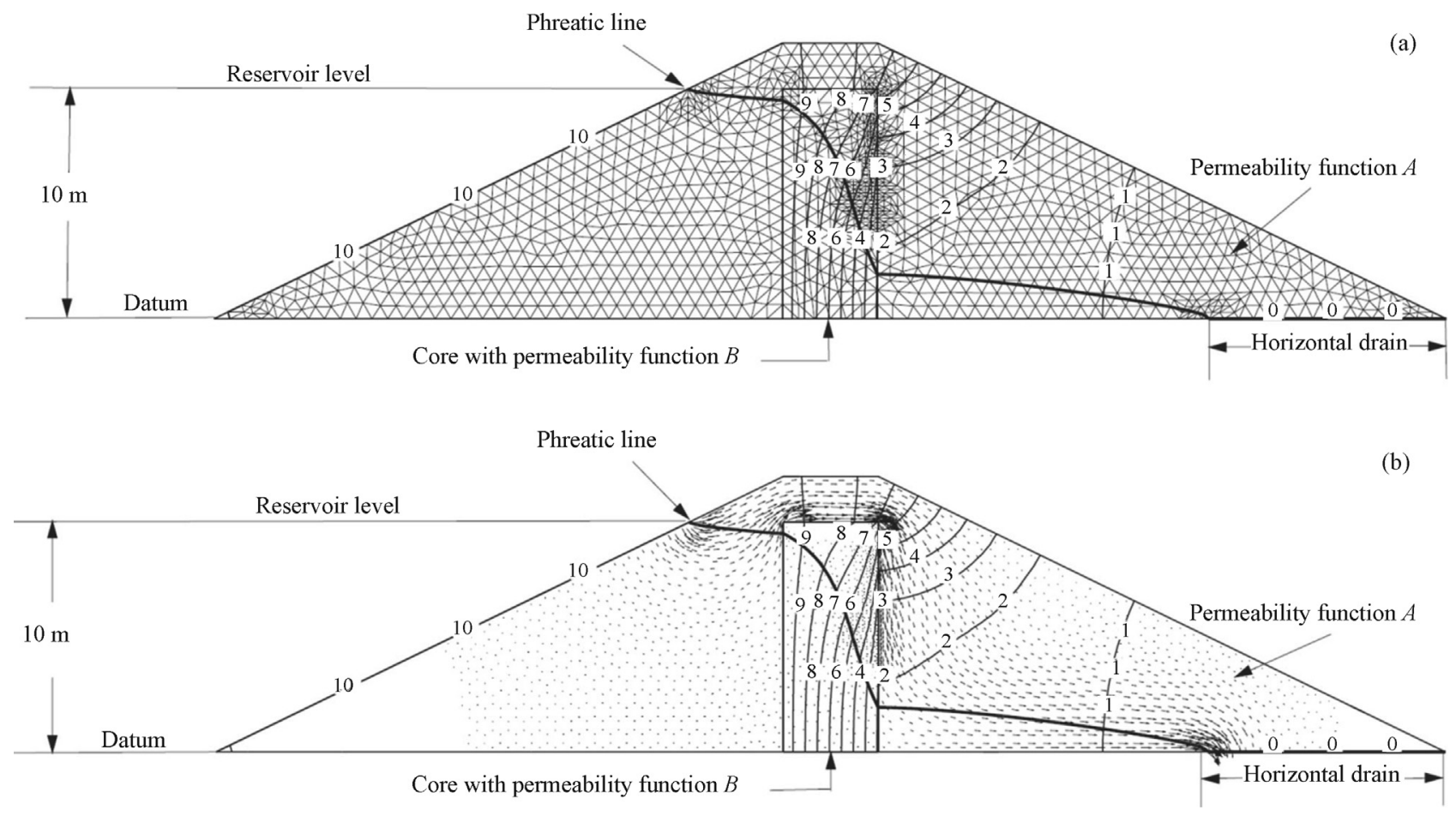

Figure 12 - a) Finite element mesh and hydraulic heads under steady state conditions. b) Velocity vectors and hydraulic heads under steady state conditions.

shows that the converged solution obtained when using the automatic adaptive mesh refinement has larger elements in most parts of the analyzed domain. The exception is around the cut-off wall where the finite element sizes are significantly smaller than the overall average element size. For the mesh shown in Fig. 13, the calculated flow rate was $6.82 \times 10^{-7} \mathrm{~m}^{3} / \mathrm{s}$ using the automatic mesh refinement code of SVFlux - FlexPDE. A comparison of seepage rate results obtained when using manually-controlled mesh and the automatic-controlled adaptive mesh are shown in Fig. 14. The 


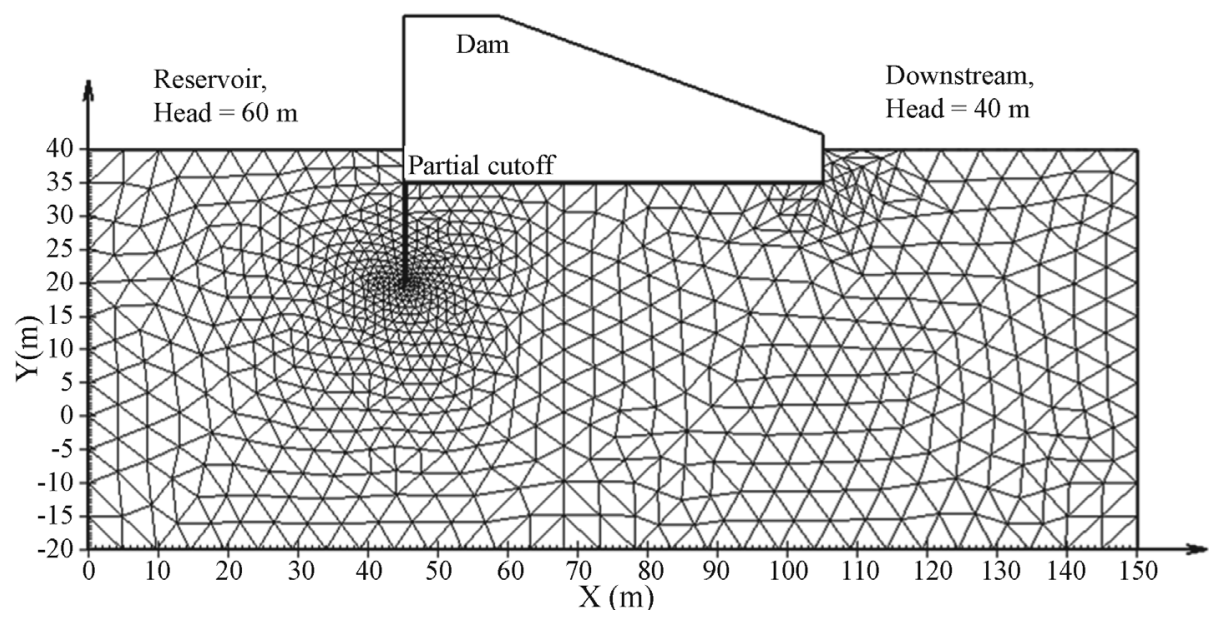

Figure 13 - Partial cut-off wall model geometry with mesh generated using the automatic adaptive mesh generator.

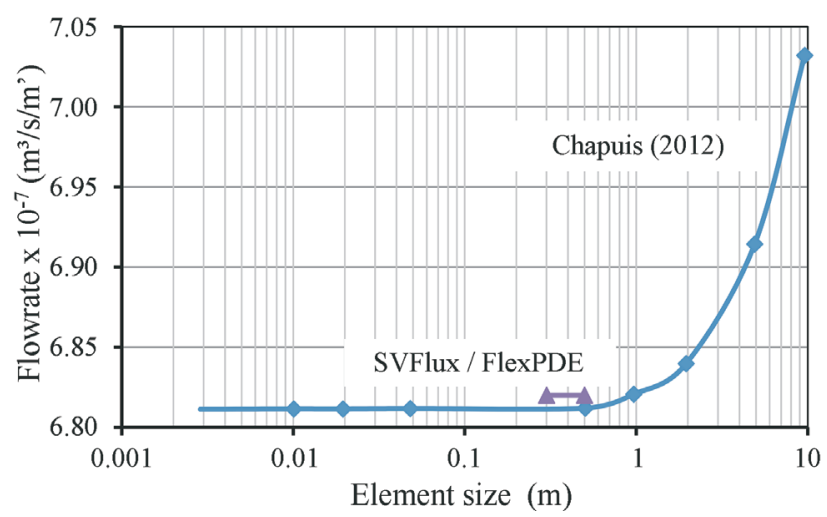

Figure 14 - Converged leakage flow-rate for the cut-off example.

automatic adaptive mesh refinement technique required considerably less computer time to obtain an accurate solution.

\subsection{Confined aquifer example}

The geometry of the second example problem is presented in Fig. 15. The pumping well is in a confined aquifer with a saturated coefficient of permeability of $4.0 \times 10^{-4} \mathrm{~m} / \mathrm{s}$.

Figure 15 shows the model geometry with the finite element mesh generated using the adaptive generator.
There are some triangular elements that have angles larger than 90 degrees and this is not the preferred shape for calculation purposes. The problem is axisymmetric with a radius of $600 \mathrm{~m}$. The converged solution when using a uniform mesh occurred with an element size of $0.1 \mathrm{~m}$ (Chapuis, 2012). The same problem was solved using automatic mesh refinement in the SVFlux - FlexPDE code. The converged solution obtained using the automatic adaptive mesh has larger elements in most parts of the analyzed domain with the exception of the region around the pumping well where the element size is significantly smaller. For the mesh presented in Fig. 15 the computed flow-rate was $369.2 \mathrm{~m}^{3} /$ day.

Figure 16 shows a comparison of the flow-rate obtained when the mesh was manually-controlled and when the mesh was automatically generated using adaptive mesh refinement. Figure 17 shows a comparison of the total head calculated at the well.

The results from the reported study indicate that the use of a finer mesh does not necessarily provide a more accurate solution. The primary concern is that the calculated result can be in error if the finite element mesh is not sufficiently refined. The results obtained when using automatic adaptive mesh refinement procedure can have larger elements over most parts of the domain while the accuracy of the solution remains preserved.

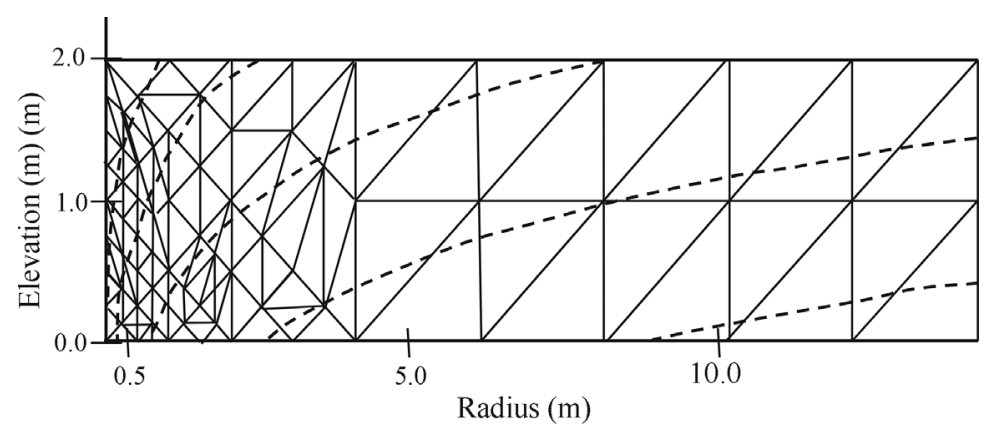

Figure 15 - Pumping well in a confined aquifer. 


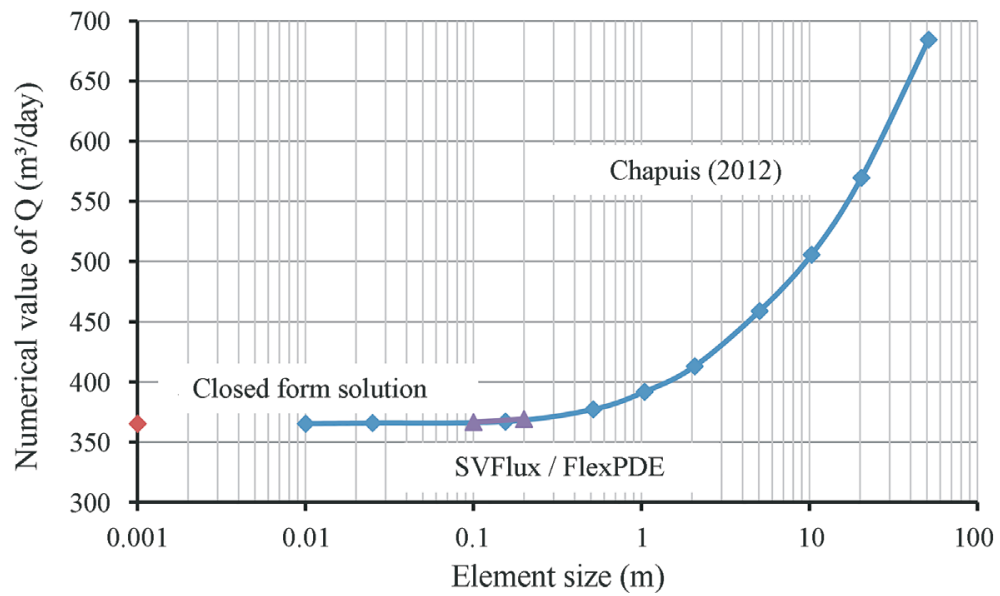

Figure 16 - Converged numerical flow-rates.

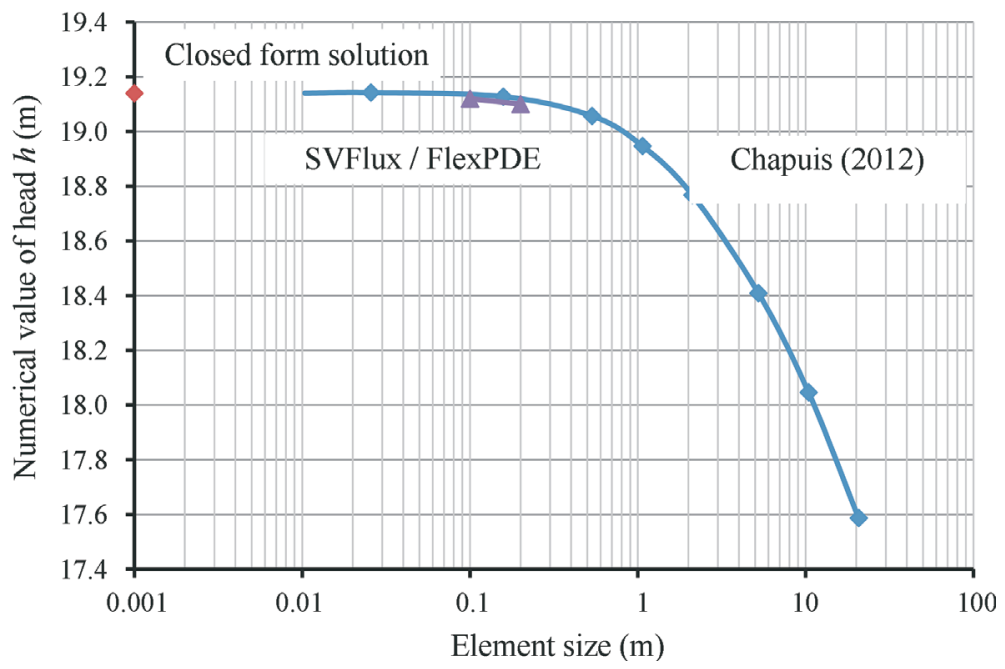

Figure 17 - Converged total heads at a radius, $r$ equal to $20.15 \mathrm{~m}$.

\section{Conclusions}

There are distinct differences in the manner in which saturated and unsaturated soils problems are solved. Unsaturated soils problems generally require the solution of a nonlinear partial differential equation because of the nonlinear nature of the soil properties. Automatic mesh refinement techniques developed in recent years for the solution of nonlinear partial differential equations are useful in ensuring that an accurate solution is obtained when unsaturated soils problems are analyzed.

One of the valuable assets of the automatic mesh refinement technique is the assurance that convergence requirements have been satisfied when solving unsaturated soils problems. The optimized mesh (i.e., use of locally finer and coarser elements) means fewer equations need to be solved and at the same time the accuracy of the solution is assured. Finite element computer codes have proven to be a valuable tool for solving the nonlinear partial differen- tial equations associated with unsaturated soils. Computers have also been useful in performing the calculations associated with the estimation of unsaturated soil property functions. Geotechnical engineers can now think in terms of simultaneously solving the saturated and unsaturated soil zones.

\section{References}

Akin, J.E. (2005). Finite Element Analysis with Error Estimators: An Introduction to the FEM and Adaptive Error Analysis for Engineering Students. Elsevier Butterfworth-Heinemann, Oxford, 512 p.

Chapuis, R.P. (2012). Influence of element size in numerical studies of seepage: Small-scale details, Geotechnical News, 30(1): 32-35.

Fredlund, D.G. \& Rahardjo, H. (1993). Soil Mechanics for Unsaturated Soils. John Wiley \& Sons, New York, 517 p. 
Fredlund, D.G.; Rahardjo, H. \& Fredlund, M.D. (2012). Unsaturated Soil Mechanics in Engineering Practice. John Wiley \& Sons, New York, 926 p.

Fredlund, D.G. \& Stianson, J. (2011). Utilization of weather station data for the assessment of ground surface moisture fluxes. Proc. Fifth Asia-Pacific Conference on Unsaturated Soils, Payatta, Thailand, pp. 1-18.

Fredlund, M.D. (2012). SVFlux User's Manual. SoilVision Systems Ltd. Saskatoon, Canada, 112 p.

Fredlund, M.D.; Zhang, J.M.; Tran, D. \& Fredlund, D.G. (2011). Coupling heat and moisture flow for the computation of actual evaporation.Proc. Canadian Geotechnical Conference and Fifth Pan-American Conference, Toronto, Paper 1058.

Oberkampf, W.L.; Blottner, F.G. \& Aeschliman, D.P. (1995). Methodology for computational fluid dynamics code verification/validation. Proc. AIAA Fluid Dynamics Conference, San Diego, Paper 95-2226.
Petrovic, I. \& Fredlund, M.D. (2012). Benefits of adaptive mesh refinement. Geotechnical News, 30(4): 37-40.

Reddy, J.N. (2006). An Introduction to the Finite Element Method. McGraw-Hill, Boston, 784 p.

Roache P.J. (2009). Fundamentals of Verification and Validation. Hermosa Publishers, Socorro, 648 p.

Terzaghi, K. (1943). Theoretical Soil Mechanics. Wiley, New York, 510 p.

Tran, Dat Tien Quoc (2013). Re-Visitation of Actual Evaporation Theories. Ph.D. Thesis, University of Alberta, Edmonton, 420 p.

Wilson, G.W. (1990). Soil Evaporative Fluxes for Geotechnical Engineering Problems. PhD Thesis, Department of Civil and Geological Engineering, University of Saskatchewan, 464 p.

Wilson, G.W.; Fredlund, D.G. \& Barbour, S.L. (1994). Coupled soil-atmospheric modeling for soil evaporation, Canadian Geotechnical Journal, 31(2):151-161.

Zienkiewicz, O.C.; Taylor, R.L. \& Zhu, J.Z. (2005) The Finite Element Method: Its Basis and Fundamentals. Elsevier Butterfworth-Heinemann, Oxford, 752 p. 